\title{
Recombinant protein subunit vaccine reduces puerperal metritis incidence and modulates the genital tract microbiome
}

\author{
E. B. S. Meira Jr., ${ }^{1}$ R. D. Ellington-Lawrence, ${ }^{1}$ J. C. C. Silva, ${ }^{1}$ C. H. Higgins, ${ }^{1}$ R. Linwood, ${ }^{2}$ M. X. Rodrigues, ${ }^{1}$ \\ L. Bringhenti, ${ }^{1}$ H. Korzec, ${ }^{1}$ Y. Yang, ${ }^{1}$ M. Zinicola, ${ }^{1}$ and R. C. Bicalho ${ }^{1 *}$ \\ ${ }^{1}$ Department of Population Medicine and Diagnostic Sciences, Cornell University, Ithaca, NY 14853-6401 \\ ${ }^{2}$ Merck Animal Health, De Soto, KS 66018
}

\begin{abstract}
The objective of this study was to evaluate the efficacy of 3 vaccine formulations containing proteins (FimH, leukotoxin, and pyolysin), inactivated whole cells (Escherichia coli, Fusobacterium necrophorum, and Trueperella pyogenes), or both, in the prevention of postpartum uterine diseases. A randomized clinical trial was conducted at a commercial dairy farm; 800 heifers were assigned into 1 of 4 different treatment groups: control, vaccine 1 (bacterin and subunit proteins), vaccine 2 (bacterin), and vaccine 3 (recombinant subunit proteins), and each heifer received a subcutaneous injection of its respective treatment at $240 \pm 3$ and $270 \pm 3 \mathrm{~d}$ of gestation. Vaccination significantly reduced the incidence of puerperal metritis when compared with control (9.1\% vs. $14.9 \%$, respectively; odds ratio 0.51 ). Additionally, vaccine 3 was found to reduce the incidence of puerperal metritis when compared with the control (8.0\% vs. $14.9 \%$, respectively; odds ratio 0.46$)$. Reproduction was improved for metritic cows that were vaccinated, and the effect was stronger for cows that were treated with vaccine 3 . In general, vaccination decreased the total vaginal bacterial load and decreased the vaginal load of $F$. necrophorum by $9 \mathrm{~d}$ in milk. Vaccination reduced the prevalence of puerperal metritis in the first lactation of dairy cows, leading to less metritic disease and improved reproduction.
\end{abstract}

Key words: metritis, Fusobacterium necrophorum, reproduction, microbiome, vaccination

\section{INTRODUCTION}

Metritis is an important disease for the dairy industry globally due to its high prevalence (25-40\%), associated economic losses, and effect on animal welfare. Metritis

Received May 22, 2019.

Accepted March 31, 2020.

*Corresponding author: rcb28@cornell.edu reduces the profit potential of dairy farms because of treatment costs, impaired reproductive performance, and increased involuntary culling (Overton and Fetrow, 2008; Sheldon et al., 2008).

Escherichia coli, Trueperella pyogenes, Prevotella melaninogenica, and Fusobacterium necrophorum, utilizing culture-dependent methods, have historically been implicated as the main bacteria associated with uterine infection (Dohmen et al., 2000; Azawi, 2008). However, recent metagenomic-based studies of the metritic bovine uterine microbiome have found relatively low abundances of Escherichia coli and Trueperella pyogenes. In contrast, a dysbiotic uterine microbiome has been associated with a higher abundance of species of the Bacteroides, Porphyromonas, and Fusobacterium genera (Jeon and Galvão, 2018). Bicalho et al. (2017b) found the highest estimated bacterial loads of Fusobacteria at 3 and 7 DIM. Bicalho et al. (2012) reported that the bacterial etiology of postpartum uterine diseases is dynamic and multifactorial. Several cow-related factors known to increase uterine diseases by facilitating bacterial contamination of the reproductive tract (e.g., assisted parturition, twins, retained placenta, and stillbirth) were associated with higher total bacterial load (TBL) and higher estimated loads of Fusobacterium and Bacteroidetes after calving. In that study, E. coli was suggested to disrupt the natural balance of the uterine microbiota, facilitating subsequent infection by Trueperella and Fusobacterium species. Therefore, species with lower relative abundances can still play an important role in the pathogenic process and should still be considered in the evaluation of novel vaccine targets. Cows infected with FimH-carrying E. coli at 1 to 3 DIM were at higher risk of developing metritis and having subsequent $F$. necrophorum intrauterine infection at 8 to 10 DIM.

Bicalho et al. (2010) found that the presence of $E$. coli virulence factor (VF) FimH was associated with metritis and endometritis. One hundred percent of T. pyogenes isolates from metritis cases were found to carry the pyolysin (plo) gene (Santos et al., 2010). 
Fusobacterium necrophorum lktA (leukotoxin) is a third VF linked to metritis and implicated in the pathogenic action of the bacterium (Bicalho et al., 2012).

Puerperal metritis is commonly treated with antibiotics; however, a top public health challenge is the widespread emergence of antibiotic-resistant pathogenic bacteria; hence, the extensive use of antibiotics in food animals is of growing concern (Dolejska et al., 2011; Aust et al., 2013). Considering this morbidity, an efficacious vaccine against metritis would improve animal welfare, reduce economic losses, potentially limit the use of antibiotics, and curb environmental shedding of resistant bacteria.

Our research group previously developed, produced, and evaluated 3 subcutaneous and 2 intravaginal vaccines containing different combinations of bacterins and specific VF proteins, including inactivated E. coli, $T$. pyogenes, and $F$. necrophorum and recombinant FimH, Plo, and Lkt proteins (Machado et al., 2014). The subcutaneous vaccines were found to be associated with a lower incidence of puerperal metritis. This study relied on culture-based methods to obtain prevalence estimates for the targeted bacteria to evaluate the vaccines' protective effects, but no differences in the prevalence of positive cultures between vaccinated and control cows were found.

We hypothesized that immunization with the aforementioned antigens delivered subcutaneously would reduce the bacterial load of the target species, thus protecting against more severe forms of uterine disease such as puerperal metritis. To date, research has shown that an individual's microbiome before immunization may interfere with vaccination efficacy in disease prevention (Brotman et al., 2014; Valdez et al., 2014). However, to our knowledge, no study has evaluated the effect of immunization on the prevalence of a specific disease associated with the vaccine's effect on the targeted microbiome. Therefore, this study aimed to evaluate the effect of vaccination with bacterin and subunit vaccines containing inactivated E. coli, T. pyogenes, and F. necrophorum, and associated VF (FimH, Plo, and Lkt) on modulation of the vaginal microbiome and reduction of the prevalence of uterine diseases in dairy cows.

\section{MATERIALS AND METHODS}

\section{Ethics Statement}

The field trial was conducted on a commercial dairy farm located near Ithaca, New York. This farm was selected because of its long working relationship with the Ambulatory and Production Medicine Clinic at Cornell University, and the trial was authorized by the farm owner, who was aware of all procedures. The research protocol was reviewed and approved by the Institutional Animal Care and Use Committee of Cornell University (protocol number 2011-0111).

\section{Farm and Management}

Pregnant Holstein heifers were enrolled from February 10, 2017, to August 3, 2017; the follow-up period continued until November 3, 2017. The farm milked 3,600 Holstein cows 3 times daily in a 100-stall rotary milking parlor. All animals were subjected to the same immunization protocol before and during the study period. At 3 mo of age, all animals were immunized with modified-live cultures of bovine rhinotracheitis virus, bovine virus diarrhea virus (types 1 and 2); parainfluenza3 virus and bovine respiratory syncytial virus, Vista 5 SQ (Merck Animal Health, Kenilworth, NJ), Clostridium Chauvoei-Septicum-Hemolyticum-NovyiTetani-Perfringens Types C \& D Bacterin-Toxoid, Covexin (Merck Animal Health), and Moraxella bovis bacterin, Piliguard Pinkeye Triview (Merck Animal Health). Animals received a booster of each vaccine 2 wk later. At $11 \mathrm{mo}$, they received another dose of the same multivalent viral vaccine previously administered. Furthermore, at d 200 of pregnancy, animals were immunized with killed viruses of bovine virus diarrhea types 1 and 2, bovine rhinotracheitis, bovine respiratory syncytial virus, and parainfluenza3 virus, Triangle 9 (Boehringer Ingelheim Vetmedica Inc., St. Joseph, MO), and another dose of the previously administered clostridial vaccine. At d 250 and 264 the heifers were immunized with an E. coli J5 bacterin, J-Vac (Merial, Duluth, GA), and a combination inactivated bovine rotavirus, bovine coronavirus, K99 E. coli bacterin, and C. perfringens type $\mathrm{C}$ toxoid vaccine, Scourguard (Zoetis, Florham Park, NJ). Finally, at 35 DIM, they were immunized with the previously used multivalent viral vaccine and $E$. coli O111:B4 bacterin, and at the first pregnancy diagnosis date, they received a final dose of the E. coli O111:B4 bacterin. The heifers were housed in freestall barns with concrete stalls covered with mattresses and bedded with manure solids. All cows were offered a TMR consisting of approximately $55 \%$ forage (corn silage, haylage, and wheat straw) and $45 \%$ concentrate (corn meal, soybean meal, canola, cottonseed, and citrus pulp) on a DM basis of the diet. The diet was formulated to meet or exceed the NRC nutrient requirements for lactating Holstein cows weighing 650 $\mathrm{kg}$ and producing $45 \mathrm{~kg}$ of $3.5 \% \mathrm{FCM}$. The reproductive management utilized a combination of Presynch (Moreira et al., 2001), and Ovsynch protocols (Pursley et al., 1995), with animals bred after heat detection 
of estrus via activity monitors. However, $25 \%$ to $30 \%$ of cows open at pregnancy diagnosis were inseminated via timed AI, using the Resynch protocol (Fricke et al., 2003; ALPRO; DeLaval, Kansas City, MO). Cornell University Ambulatory Service diagnosed pregnancy 35 $\mathrm{d}$ after insemination and confirmed diagnosis around 60 $\mathrm{d}$ after insemination.

\section{Vaccine Formulation}

All vaccines were prepared at the Merck Animal Health (MAH) R and D facility in DeSoto, Kansas, based on the Machado et al. (2014) description. All vaccine components were of MAH origin. Three different subcutaneous vaccine formulations and a placebo were made: vaccine 1 was formulated with inactivated $E$. coli (12714-2), E. coli (4612-2), T. pyogenes (10481-8), T. pyogenes (6375-1), F. necrophorum (5663), F. necrophorum (513), recombinant FimH pili protein, recombinant pyolysin protein, and leukotoxin (FimH, Plo, and Lkt) antigens and blended with aluminum hydroxide; vaccine 2 was composed of inactivated E. coli (12714-2), T. pyogenes (10481-8) and F. necrophorum (5663) antigens, and blended with aluminum hydroxide; vaccine 3 was formulated with recombinant subunit proteins FimH, Plo, and Lkt; and a placebo, administered to a control group, contained aluminum hydroxide but no bacteria or subunit protein antigens. All vaccine components were tested for sterility by $\mathrm{MAH}$ before the final vaccine was assembled and bottled. Sterility of vaccines and endotoxin assessment were also performed by $\mathrm{MAH}$ before animal enrollment. Exogenous endotoxin levels were $<20 \mathrm{EU} / \mathrm{mL}$ for recombinant subunit vaccines and $<500 \mathrm{EU} / \mathrm{mL}$ for inactivated vaccines.

\section{Treatment Groups and Case Definition}

Heifers were chosen as experimental units because primiparous cows are at increased odds of developing metritis (Giuliodori et al., 2013). We considered a sample size of 200 heifers per group sufficient to detect a 10-percentage-point reduction in the incidence of puerperal metritis (from 20\% in control to $10 \%$ in vaccinated animals). Sample size was determined assuming a desired type I error rate of $5 \%$, and a statistical power of $80 \%$. Animals enrolled in the study were randomly allocated into 1 of 4 treatment groups using the random number function of Excel (Microsoft Corp., Redmond, WA). Pregnant heifers, days carried calf of $240 \pm 3$, were enrolled in the study weekly in blocks of 40 cows. A subset of cows was assigned for sample collection of vaginal swabs (VS) and were randomly selected a priori to ensure that the data would be balanced be- tween treatment groups as well as enrollment dates. In total, 800 pregnant heifers were enrolled in the study; 200 were randomly allocated to vaccine 1 , vaccine 2 , vaccine 3 , or control. Heifers received 2 doses of their respective treatments by the research group at $240 \pm 3$ and $270 \pm 3 \mathrm{~d}$ of pregnancy. Heifers that calved before the expected due date and could not receive both treatments, vaccination and booster, were eliminated from the study.

Information regarding ease of calving was gathered by farm workers, and a 5-point scale was used: ease 1 was defined as a calving that occurred easily without assistance; ease 2 was defined as unassisted, but more difficult than ease 1 ; ease 3 was defined as a calving requiring easy assistance from a person; ease 4 was defined as a vaginally delivered calving requiring the calf position to be corrected or hard traction to be applied to deliver the calf; and ease 5 was defined as a calving requiring fetotomy or cesarean section. Dystocia was defined as a calving with ease greater than 2 .

As part of routine fresh cow health management, trained farm personnel would follow a specific diagnostic protocol (designed by veterinarians from Cornell University's Ambulatory and Production Medicine Clinic) to diagnose and treat retained placenta, puerperal metritis, ketosis, and clinical mastitis. After parturition, cows were kept in the same pen until around 20 DIM. This pen was monitored by farm employees, and cows were submitted to a complete physical exam if they were showing signs of dullness and depression; cows with fetid, watery, red-brown uterine discharge accompanied with fever were diagnosed with puerperal metritis and treated with a subcutaneous injection of ceftiofur crystalline free acid $(6.6 \mathrm{mg} / \mathrm{kg}$, at $200 \mathrm{mg} /$ $\mathrm{mL}$ ) by farm employees. Farm personnel were blinded to the study treatments and diagnosis, the research group did not interfere with farm routine nor shared study information. The research group did not personally administer any antibiotics, and was only present on the farm to administer vaccines or placebo, and diagnose puerperal metritis, clinical metritis (CM), and purulent vaginal discharge (PVD) for the study.

Puerperal metritis diagnosis by the research team (RDPM) was performed at 3 and 7 DIM. Puerperal metritis was defined as the presence of fetid, watery, redbrown uterine discharge and rectal temperature greater than $39.5^{\circ} \mathrm{C}$ (Sheldon et al., 2006). Clinical metritis was defined as the presence of fetid, watery, red-brown uterine discharge, without fever, or rectal temperature under $39.5^{\circ} \mathrm{C}$. Rectal temperature was measured at 3 and 7 DIM using a digital thermometer (GLA M750, GLA Agricultural Electronics, San Luis Obispo, CA) equipped with an angle probe $\left(11.5 \mathrm{~cm}, 42^{\circ}\right)$. Retained 
placenta was defined as a condition where cows failed to release their fetal membranes within $24 \mathrm{~h}$ of calving (Kelton et al., 1998). Vaginal tears were defined as vulvovaginal lacerations greater than $2 \mathrm{~cm}$ at the dorsal commissure of the vulva, the lateral walls of the vulva/vagina, or both (Vieira-Neto et al., 2016); the research team would evaluate vaginal lacerations by visual inspection on d 3 and 7 postpartum. Clinical forms used to take notes on observations would not have any information regarding experimental treatment, to guarantee that observers were blinded at the time of health assessment. Information regarding metritis diagnosis was not exchanged between farm personnel and the research team. Data regarding health traits and reproduction were extracted from the farm's DairyComp 305 database (Valley Agricultural Software, Tulare, CA) at the end of the trial and only then were the farm data compared with the data collected by the research team.

Diagnosis of PVD was performed at $25 \pm 3$ DIM and $45 \pm 3$ DIM by visual examination of the vaginal contents using the Metricheck device (Metricheck, SimcroTech, Hamilton, New Zealand). Vaginal discharge was scored using a 0 to 3 scale: $0=$ clear mucus, 1 $=$ flecks of pus in the vaginal discharge, $2=<50 \%$ of pus in the vaginal discharge, $3=>50 \%$ of pus in the vaginal discharge. Animals with scores of $\geq 2$ were considered as positive for PVD (Sheldon et al., 2006).

\section{Collection of Microbiome Samples}

Thirty heifers from each treatment were randomly selected for microbiome investigation. These groups included some heifers that were treated with antibiotics by farm workers. Vaginal swabs were collected from a randomly selected subset of cows (30/treatment) at 3 , 7 , and 9 DIM. Cows were restrained and the perineum area was cleansed and disinfected with $70 \%$ ethanol solution. The vulva labia were opened, and the swab was applied to a single site at the midpoint of the vaginal cavity, swirling it 6 times, exposing it to genital secretion, and then withdrawing it without contamination. The swab samples were transported to the laboratory on ice and frozen at $-80^{\circ} \mathrm{C}$ until processing. Due to ongoing losses in the dairy herd, only 103 cows were followed through the entire course of the postpartum period across the treatment and control groups, which yielded 260 samples from across 3, 7, and 9 DIM. Exclusion of animals due to antibiotics administration by farm workers ( $\mathrm{n}=10$ cows removed) reduced usable DNA swabs from 260 to 237 swabs (23 swabs removed from across a total of 10 cows). Out of 93 animals in the final group, 3 animals were missing samples from 2 time points and 36 animals were missing a sample from one time point due to collection failure.

\section{DNA Extraction}

The DNA was isolated from 237 VS by adding 1.5 $\mathrm{mL}$ of DNA-free water into 2-mL microcentrifuge tubes that contained individual sample swabs. Particulate matter was dislodged from the swab through agitation by vortex with a vortex adapter (Qiagen, Hilden, Germany). The swabs were aseptically removed from the microcentrifuge tubes, and the remaining liquid was centrifuged at $10,000 \times g$ for $10 \mathrm{~min}$ at $21^{\circ} \mathrm{C}$. The supernatant was discarded, and the DNA was extracted from the pellet using the PowerFood DNA isolation kit (Qiagen) according to the manufacturer's specifications. Concentration and purity of DNA were evaluated using a NanoDrop ND-1000 spectrophotometer (NanoDrop Technologies, Rockland, DE) at wavelengths of 230, 260, and $280 \mathrm{~nm}$. Elution buffer from the PowerFood DNA isolation kit was used as a blank and $2 \mu \mathrm{L}$ of the extracted DNA solution was measured. Extracted DNA samples were stored at $-20^{\circ} \mathrm{C}$.

\section{PCR and Sequencing}

The 16S amplicons were amplified by PCR for individual metagenomic DNA samples according to previously described methodology (Bicalho et al., 2017b). The 237 samples were individually tagged with a unique 12-bp error-correcting Golay barcode primer picked from the Earth Microbiome Project (http:// www.earthmicrobiome.org/; Gilbert et al., 2010). The V4 hypervariable region of bacterial/archaeal $16 \mathrm{~S}$ rRNA genes was amplified with barcoded $515 \mathrm{~F}$ and $806 \mathrm{R}$ primers using methods optimized for the Illumina MiSeq platform (Caporaso et al., 2012).

The 5'-barcoded amplicons for each vaginal sample were generated in triplicate in 96-well plates (VWR) using 12 to $300 \mathrm{ng}$ of template DNA, $1 \times$ EconoTaq Plus Green Master Mix (Lucigen, Middleton, WI), and $10 \mu M$ of each primer. The PCR of the $16 \mathrm{~S}$ rRNA gene used an initial denaturing step of $94^{\circ} \mathrm{C}$ for $3 \mathrm{~min}$, followed by 35 cycles of $94^{\circ} \mathrm{C}$ for $45 \mathrm{~s}, 50^{\circ} \mathrm{C}$ for $1 \mathrm{~min}$, and $72^{\circ} \mathrm{C}$ for $90 \mathrm{~s}$, and then a final elongation step of $72^{\circ} \mathrm{C}$ for $10 \mathrm{~min}$. Following the reaction, samples were maintained at $-20^{\circ} \mathrm{C}$ until purification. Before sequencing, the replicate amplicons for each sample were pooled and purified and visualized by electrophoresis through $1.2 \%$ (wt/vol) agarose gels stained with $0.5 \mathrm{mg} / \mathrm{mL}$ ethidium bromide. Blank primer controls, which contained no DNA, were included in each gel run. In all cases the blank controls produced no visible PCR products and all samples were included in further analysis. Purified amplicon DNA was quantified using Quant-iT PicoGreen and a dsDNA Broad Range Assay Kit (Life Technologies Corporation, Carlsbad, CA). 


\section{Real-Time Quantitative PCR}

Quantitative PCR (qPCR) was conducted to determine the TBL and the absolute numbers of fim $H$, $p l o$, and lkt, known putative VF genes of E. coli, T. pyogenes, and F. necrophorum, respectively. Across the 3 treatment groups and the control group, 237 samples were successfully processed (23 cows/treatment \pm 2 ; vaccine $1=61$ samples, vaccine $2=61$ samples, vaccine $3=56$ samples, control $=59$ samples).

A plasmid containing the amplified V4 hypervariable region and the amplified VF genes was cloned to create a standard curve. The $16 \mathrm{~S}$ rRNA gene copy numbers were quantified by qPCR using Unibac forward and reverse primers. The PCR were performed in $15 \mu \mathrm{L}$ volumes composed of 1X iQTMSybr Green Supermix (Bio-Rad Laboratories, Hercules, CA), $300 \mathrm{nM}$ each primer and $50 \mathrm{ng}$ to $5 \mathrm{pg}$ of the genomic DNA (or plasmid DNA standards). Quantification of the $16 \mathrm{~S}$ rRNA gene and VF genes was achieved by using serial 10-fold dilutions from $10^{0}$ to $10^{6}$ plasmid copies of the previously quantified plasmid standards. Plasmid standards and samples were run in duplicates. The average of the cycle threshold value was used to calculate the estimated TBL and the bacterial load of each target gene as previously described by Bicalho et al. (2017b).

\section{Sequencing and Bioinformatics}

We performed 3 sequencing runs using the MiSeq sequencer (Illumina Inc., San Diego, CA) and v2 reagent kits (300 cycles). The purified amplicons were quantified and standardized to the same concentration using a Qubit 3.0 fluorometer (Thermo Fisher Scientific), and sequencing was performed on the Illumina MiSeq platform (Illumina Inc.) using the MiSeq reagent kit v2 at 300 cycles. No samples failed to amplify and all were sequenced. The final Illumina sequenced data set contained 237 samples from 93 cows from 3, 7 , and 9 DIM (23 cows/treatment \pm 3 ; vaccine $1=61$ samples, vaccine $2=61$ samples, vaccine $3=56$ samples, control $=59$ samples). We processed the generated $16 \mathrm{~S}$ rDNA sequences by using the MiSeq Reporter Metagenomics Workflow (Illumina Inc.), which is a pipeline based in the Greengenes database (http://greengenes.lbl.gov) and provides output as classifications by total reads and relative abundance at multiple taxonomic levels: kingdom, phylum, class, order, family, genus, and species. This was used to obtain pertinent taxonomic data. Community structure was analyzed using the open-source software pipeline Quantitative Insights into Microbial Ecology (QIIME) version 1.7.0-dev. Sequences were filtered for quality using previously established guidelines. Then the sequences were binned into final operational taxonomic units (OTU) based on the Greengenes Reference Database, May 2013 release. All low-abundance clusters were filtered and chimeric sequences were removed using USEARCH. Representative sequences for each OTU were matched against the Greengenes database and only full-length, high-quality reads were used for analysis. From the matched OTU, Shannon index, and Chao 1 index outputs were generated. Before the estimation of the Shannon and Chao 1 indexes, all sample libraries were rarefied to an equal depth of 10,000 reads using QIIME. Following the quality control steps of the QIIME pipeline, 173 samples from 3,7 , and 9 DIM were used to examine the microbiome diversity results.

\section{Statistical Analysis}

The incidence of risk factors for metritis (retained placenta, stillbirth, dystocia, and vaginal tears) was compared among groups with one-way nonparametric ANOVA tests in JMP Pro 13 (SAS Institute Inc., Cary, NC) software. To assess the effect of treatment group on the probability of the outcome variables (prevalence at $3,7,25$, and 45 DIM and cumulative incidence by 7 DIM, or by 20 DIM), a mixed logistic regression model was fitted to the data for each one of the disease outcome variables in SAS using the logistic procedure. Contrasts were performed to compare the effect of vaccination (vaccine 1 , vaccine 2 , vaccine 3 , or any vaccine) versus control. Reproduction was measured by days open and analyzed by the estimation of the conception hazard ratio. The effect of vaccination on time to pregnancy was analyzed with multivariable survival analysis using Cox's proportional hazard regression procedure in SAS; the effect of metritis was forced into the model.

Using the total number of reads and the classification data obtained from the MiSeq bioinformatics pipeline, the relative abundance of each microbial phylum within the VS was determined. For each sample, the total relative abundance $(\%)$ of each phylum was determined by the $16 \mathrm{~S}$ rDNA read count for each individual phylum compared with the sample's total read count. The mean relative abundance for each phylum across all samples was determined and all phyla that had a mean count above $>1 \%$ prevalence were considered in further data analysis; all others were merged into a phylum category called "others." This process was also repeated to determine relative abundance at the species level.

A mixed model with 2-way repeated-measures ANOVA tests was fitted to the data using the standard least squares means with REML emphasis in JMP Pro 13 (SAS Institute Inc.) software. The effects of vaccination 
(vaccine 1 , vaccine 2 , vaccine 3 , or any vaccine) on the dependent variables (bacterial relative abundance at the phylum and species levels, total bacterial gene copies, and species bacterial gene copies) were each evaluated separately. Time (3, 7, and 9 DIM), each independent variable, and their interactions were added as fixed effects for each respective model. The identification number of each cow was included in the analysis as a random effect to account for repeated measures. A Student's t-test or one-way ANOVA, where appropriate, were used as post-hoc tests within the mixed model to explore data interaction at specific time points. For all data, differences with a value of $P<0.05$ were considered significant and differences with a value of $P<$ 0.10 were reported as trends. Graphs were built using GraphPad Prism 7 software (GraphPad Software Inc., La Jolla, CA).

\section{RESULTS}

\section{Descriptive Statistics}

In total, 800 primiparous heifers were enrolled in the study and randomly allocated to 4 treatment groups composed of 200 heifers per group. One hundred twenty-nine heifers were excluded from the trial due to the impossibility of following up their uterine health on the first 45 d postpartum, which included heifers culled for different reasons (e.g., left displaced abomasum, mastitis, lameness, behavior, low production, ketosis) during the trial $(\mathrm{n}=85)$, calving before receiving the second vaccine dose $(\mathrm{n}=44)$, or both; no intergroup difference was observed in the proportion of excluded animals $(P$ $=0.88)$. The final distribution of the number of heifers per treatment group is presented in Table 1.

The incidence of known, naturally occurring risk factors for metritis was evenly balanced among the treatment groups (Table 1).

\section{Effects of Vaccination on the Prevalence and Cumulative Incidence of RDPM and Farm-Diagnosed Puerperal Metritis}

The effects of vaccination on the prevalence and cumulative incidence of RDPM by 7 DIM are presented in Table 2. There were no differences in the prevalence of RDPM at 3 DIM $(P=0.20)$. There was a tendency for a reduction on RDPM prevalence for animals treated with vaccine 3 at 7 DIM when compared with control ( $5.1 \%$ vs. $9.7 \%$, respectively; $P=0.08$ ), and there was a tendency for a decrease in the prevalence for vaccinated cows (any vaccine) and the prevalence of RDPM for all vaccinated animals compared with the control $(5.9 \%$ vs. $9.7 \%$, respectively; $P=0.06$ ). Vaccine 3 reduced the cumulative incidence of RDPM by 7 DIM when compared with the control $(8.0 \%$ vs. $14.9 \%$, respectively; $P$ $<0.05)$. Vaccine 1 or vaccine 2 (or both) did not cause any significant decrease in the cumulative incidence of puerperal metritis. However, when a contrast was done and the cumulative incidence of RDPM by 7 DIM of all vaccinated animals was compared with the control, vaccination reduced the incidence of puerperal metritis ( $9.1 \%$ vs. $14.9 \%$, respectively; $P=0.03)$.

According to the data extracted from the farm's DairyComp 305 database (Valley Agricultural Software, Tulare, CA), treatment of metritic cows ranged from 0 to 20 DIM and was concentrated around 7 DIM. The effects of vaccination on the incidence of farmdiagnosed puerperal metritis (FDPM) by 20 DIM are presented in Table 3. Compared with the control, vaccine 3 reduced the incidence of FDPM by 20 DIM ( $13.7 \%$ vs. $8.3 \%$, respectively; $P<0.05)$.

\section{Effect of Vaccination on the Prevalence of CM}

Clinical metritis prevalence at 3 DIM was 3.2, 1.3, $1.8,2.1$, and $1.9 \%$ for vaccine 1 , vaccine 2 , vaccine 3 ,

Table 1. Incidence of retained placenta (RP), stillbirth, dystocia, vaginal tear, and high-risk heifers in the experimental groups (vaccines $1,2,3$, and control) ${ }^{1}$

\begin{tabular}{lccccccc}
\hline & \multicolumn{9}{c}{ Incidence $(\%)$} & & \multicolumn{2}{c}{ Chi-square test } \\
\cline { 2 - 5 } \cline { 7 - 8 } Item & $\begin{array}{c}\text { Vac. } 1 \\
(\mathrm{n}=166)\end{array}$ & $\begin{array}{c}\text { Vac. } 2 \\
(\mathrm{n}=168)\end{array}$ & $\begin{array}{c}\text { Vac. 3 } \\
(\mathrm{n}=171)\end{array}$ & $\begin{array}{c}\text { Control } \\
(\mathrm{n}=166)\end{array}$ & & $\chi^{2}$ & $P$-value \\
\hline RP & 5.4 & 7.4 & 8.8 & 7.2 & & 1.72 & 0.63 \\
Stillbirth & 12.3 & 11.6 & 11.4 & 8.7 & & 1.47 & 0.69 \\
Dystocia & 31.0 & 33.3 & 31.6 & 25.6 & & 3.02 & 0.39 \\
Vaginal tear & 19.8 & 17.5 & 19.7 & 20.0 & & 0.52 & 0.91 \\
High risk & 41.7 & 46.0 & 45.1 & 43.6 & & 0.81 & 0.85
\end{tabular}

${ }^{1}$ High risk $=$ heifers that presented retained placenta, stillbirth, dystocia, or vaginal tear (or a combination of these); Vac. 1 = bacterin and subunit protein antigens; Vac. $2=$ bacterin; Vac. $3=$ subunit protein antigens; control $=$ placebo. 
Table 2. The effect of treatment (vaccines 1, 2, 3, any vaccine, and control) on the prevalence at 3 and 7 DIM, and the cumulative incidence of puerperal metritis at 7 DIM $^{1}$

\begin{tabular}{|c|c|c|c|}
\hline Variable & $\begin{array}{l}\text { Puerperal } \\
\text { metritis } \\
(\%)\end{array}$ & $\begin{array}{c}\text { Odds ratio } \\
(95 \% \text { CI })\end{array}$ & $P$-value \\
\hline \multicolumn{4}{|l|}{$3 \mathrm{DIM}$} \\
\hline Vaccine 1 & 3.2 & $0.58(0.17-1.97)$ & 0.39 \\
\hline Vaccine 2 & 2.6 & $0.52(0.14-1.89)$ & 0.32 \\
\hline Vaccine 3 & 2.5 & $0.50(0.14-1.80)$ & 0.29 \\
\hline Control & 4.5 & Baseline & \\
\hline \multicolumn{4}{|l|}{ Contrast } \\
\hline Vaccine $1+2+3$ & 2.7 & $0.53(0.20-1.41)$ & 0.21 \\
\hline \multicolumn{4}{|l|}{7 DIM } \\
\hline Vaccine 1 & 6.6 & $0.55(0.22-1.36)$ & 0.19 \\
\hline Vaccine 2 & 6.1 & $0.54(0.21-1.37)$ & 0.19 \\
\hline Vaccine 3 & 5.1 & $0.43(0.16-1.10)$ & 0.08 \\
\hline Control & 9.7 & Baseline & \\
\hline \multicolumn{4}{|l|}{ Contrast } \\
\hline Vaccine $1+2+3$ & 5.9 & $0.56(0.29-1.08)$ & 0.06 \\
\hline \multicolumn{4}{|l|}{3 and 7 DIM } \\
\hline Vaccine 1 & 10.1 & $0.54(0.26-1.13)$ & 0.10 \\
\hline Vaccine 2 & 9.1 & $0.55(0.26-1.17)$ & 0.12 \\
\hline Vaccine 3 & 8.0 & $0.46(0.21-1.00)$ & $<0.05$ \\
\hline Control & 14.9 & Baseline & \\
\hline \multicolumn{4}{|l|}{ Contrast } \\
\hline Vaccine $1+2+3$ & 9.1 & $0.51(0.29-0.93)$ & 0.03 \\
\hline
\end{tabular}

${ }^{1}$ Vaccine $1(\mathrm{n}=166)=$ bacterin and subunit protein antigens; vaccine $2(\mathrm{n}=168)=$ bacterin; vaccine $3(\mathrm{n}=171)=$ subunit protein antigens; vaccine $1+2+3(\mathrm{n}=505)=$ any vaccine; control $(\mathrm{n}=166)$ $=$ placebo.

any vaccine, and control, respectively. The prevalence of $\mathrm{CM}$ at 7 DIM was 11.3, 11.4, 9.5, 10.7, and $6.8 \%$ for vaccine 1 , vaccine 2 , vaccine 3 , any vaccine, and control, respectively. The cumulative incidence by 7 DIM was $14.1,12.5,11.2,12.6$, and $8.7 \%$ for vaccine 1 , vaccine 2 , vaccine 3 , any vaccine, and control, respectively. No difference between groups was observed in the prevalence of CM diagnosed at 3 DIM, 7 DIM, and the cumulative incidence of CM by 7 DIM when evaluated separately or when grouped.

\section{Effects of Vaccination on the Prevalence of PVD}

Vaccines were not effective in preventing PVD; no difference was observed in the prevalence of PVD between groups at 25 and 45 DIM, when evaluated separately, or when grouped. The prevalence of PVD at 25 DIM was $45.8,44.6,37.1,42.5$, and $40.4 \%$ for vaccine 1 , vaccine 2 , vaccine 3 , any vaccine, and control, respectively. The prevalence at 45 DIM was 2.6, 2.0, 4.5, 3.1, and $5.3 \%$ for vaccine 1 , vaccine 2 , vaccine 3 , any vaccine, and control, respectively.

\section{Effect of Vaccination on Reproduction}

Vaccinated cows had 4.1 higher hazard to conceive than controls (any vaccine vs. control, 95\% CI 1.2-14.1,
$P=0.02)$. Cows that received vaccine 3 had 2.7 times higher hazards to conceive than controls (vaccine 3 vs. control, 95\% CI 1.0-6.9, $P<0.05)$.

\section{Effects of Vaccination on Bacterial Community Diversity}

In the postpartum period, the Shannon and Chao 1 indexes of vaccinated cows and control cows significantly fluctuated over time (Shannon index, $P<0.01$; Chao 1, $P<0.01$ ), but no difference was observed in $\alpha$ diversity between cows treated with vaccine 1 , vaccine 2 , or vaccine 3 compared with the control cows (Shannon index, $P=0.64$; Chao $1, P=0.90$; Figure 1 ).

\section{Effects of Vaccination on the Relative Abundance of Bacterial Phyla}

The relative abundance of vaginal microbial taxa at 3,7 , and 9 DIM was described at the phylum level. The most prevalent phyla were Fusobacteria, Bacteroidetes, Firmicutes, Tenericutes, Proteobacteria, and Actinobacteria, with mean abundances ( \pm SEM) across all samples of $31.1 \pm 1.1 \%, 21.9 \pm 1.0 \%, 19.1 \pm 0.9 \%, 16.1 \pm 1.3 \%$, $10.3 \pm 0.8 \%$, and $1.0 \pm 0.1 \%$, respectively. All phyla with a relative abundance of less than $1 \%$ were excluded from the analysis. The relative abundances of bacterial phyla of vaccinated cows were similar for vaccine 1 , vaccine 2 , or vaccine 3 treated cows when individually compared with the control group (Supplemental Figure S1; https://doi.org/10.3168/jds.2019-17006). When the relative abundance of Bacteroidetes was compared between vaccinated cows and control cows, over time relative abundance between the 2 groups significantly differed $(P=0.02)$. From 3 to 7 DIM, vaccinated cows and control cows had similar abundances of Bacteroidetes (3 DIM, $P=0.20$; 7 DIM, $P=0.12$ ); however, at 9 DIM control cows had a tendency to have a higher

Table 3 . The effect of treatment (vaccines $1,2,3$, any vaccine, and control) on the incidence of puerperal metritis diagnosed by farm personnel by $20 \mathrm{DIM}^{1}$

\begin{tabular}{lccr}
\hline Variable & $\begin{array}{c}\text { Metritis } \\
(\%)\end{array}$ & $\begin{array}{c}\text { Odds ratio } \\
(95 \% \mathrm{CI})\end{array}$ & $P$-value \\
\hline Vaccine 1 & 11.2 & $0.68(0.32-1.44)$ & 0.31 \\
Vaccine 2 & 14.1 & $0.99(0.48-2.05)$ & 0.99 \\
Vaccine 3 & 8.3 & $0.44(0.19-0.99)$ & $<0.05$ \\
Control & 13.7 & Baseline & \\
Contrast & & & \\
Control & 13.7 & Baseline & \\
Vaccine $1+2+3$ & 11.2 & $0.68(0.37-1.25)$ & 0.19 \\
\hline
\end{tabular}

${ }^{1}$ Vaccine $1(\mathrm{n}=166)=$ bacterin and subunit protein antigens; vaccine $2(\mathrm{n}=168)=$ bacterin; vaccine $3(\mathrm{n}=171)=$ subunit protein antigens; vaccine $1+2+3(\mathrm{n}=505)=$ any vaccine; control $(\mathrm{n}=166)$ $=$ placebo. 

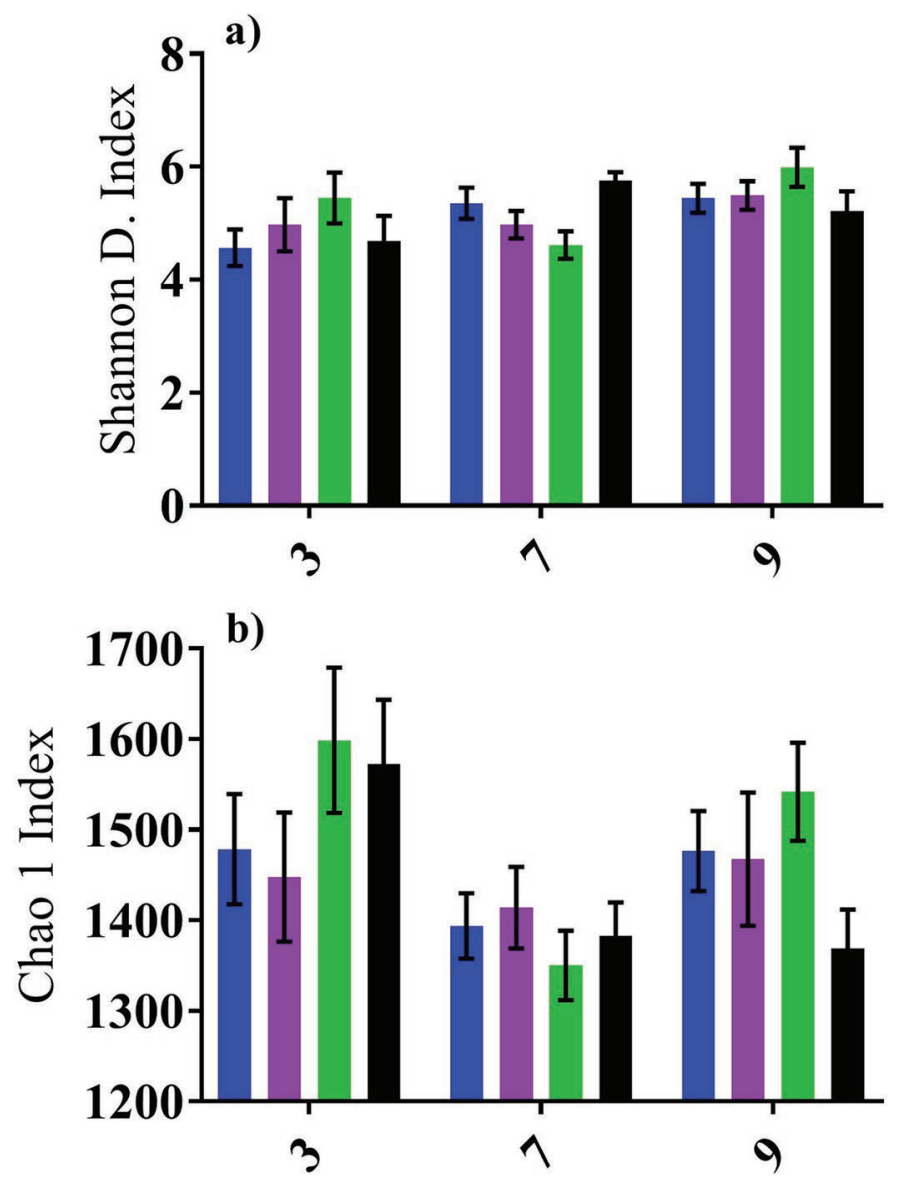

Days relative to parturition

Figure 1. Estimation of the Shannon diversity (D.) index (a) and Chao 1 index (b) for the vaginal microbiomes of dairy cows at 3,7 , and 9 DIM. Data are presented as LSM \pm SEM ( $n=173$ swabs). Graphs compare the effect of individual vaccines $(\mathrm{V} 1=$ blue, $\mathrm{V} 2=$ purple, or $\mathrm{V} 3=$ green) and control on $\alpha$ diversity over time.

relative abundance of Bacteroidetes than vaccinated cows (9 DIM, $P=0.07$; Supplemental Figure S1; https: //doi.org/10.3168/jds.2019-17006). The relative abundance for the other phyla remained similar over time, except for Proteobacteria $(P=0.01)$ and Firmicutes $(P$ $<0.01$ ), which significantly fluctuated over time in the postpartum period (Supplemental Figure S1).

\section{Effects of Vaccination on the Relative Abundance of Bacterial Species}

The relative abundance of vaginal taxa at 3,7 , and 9 DIM was also described at the species level. All species $(\mathrm{n}=12)$ with a mean relative abundance greater than $1 \%$ are described in Supplemental Figure S2 (https:// doi.org/10.3168/jds.2019-17006). The 5 most abundant species were Fusobacterium necrophorum, Sneathia sanguinegens, Ureaplasma diversum, Porphyromonas levii, and Gallibacterium melopsittaci with mean relative abundances of $18.0 \pm 1.0 \%, 13.4 \pm 1.0 \%, 10.8 \pm 1.2 \%$, $5.3 \pm 0.6 \%$, and $7.0 \pm 0.5 \%$, respectively. In general, the relative abundances of vaccinated cows and control cows were similar at the species level; these results are summarized in Supplemental Figure S2.

\section{Estimated TBL Using qPCR, and the Effects of Vaccination on Estimated Species-Specific Loads}

The mean TBL for the 93 cows analyzed was 3.85 $\log _{10} 16 \mathrm{~S}$ rDNA copies \pm 0.11 (SEM). The combined mean TBL for all cows at 3, 7, and 9 DIM was $3.61 \pm$ $0.17,4.20 \pm 0.20$, and $3.74 \pm 0.22$, respectively, across all treatment groups and the control group. As shown in Figure 2a and Figure 2b, the interactions between TBL and vaccination status significantly fluctuated over time $(P=0.03)$. At 3 DIM and 7 DIM, the bacterial loads for vaccinated cows and control cows were similar (3 DIM, $P=0.92$; DIM, $P=0.49$ ). However, at 9 DIM, cows vaccinated with vaccine $1(3.16 \pm 0.38)$ or vaccine $2(3.35 \pm 0.43)$ had similarly decreased TBL compared with the vaccine $3(3.63 \pm 0.43)$ treated cows and the control group $(4.78 \pm 0.43$; vaccine $1, P=0.01$; vaccine $2, P=0.02$; vaccine $3, P=0.06$; Figure $2 \mathrm{a}$ ). Considered together, vaccinated cows had significantly decreased TBL compared with control cows at 9 DIM $(P<0.01 ;$ Figure 2b).

The lower TBL of vaccinated cows at 9 DIM was primarily driven by decreased bacterial loads of $\mathrm{Fu}$ sobacterium necrophorum (Figure 3b). The effect of vaccination status on the load of $F$. necrophorum significantly fluctuated over time $(P=0.03$; Figure $3 \mathrm{~b})$. The bacterial loads were similar for vaccinated cows and control cows at 3 and 7 DIM. However, at 9 DIM, cows vaccinated with vaccine 1 , vaccine 2 , or vaccine 3 tended to have lower bacterial loads of $F$. necrophorum compared with control cows (vaccine 1 , $P=0.03$; vaccine $2, P=0.06$; vaccine $3, P=0.09$; Figure 3a). Most importantly, when vaccinated cows as a whole were contrasted against the control group at 9 DIM, all vaccinated cows had lower bacterial loads of $F$. necrophorum compared with control cows $(P<0.01$; Figure $3 \mathrm{~b}$ ).

In addition, the bacterial load of Bacteroides heparinolyticus was statistically different over time between vaccinated cows and control cows in the postpartum period (B. heparinolyticus, $P<0.01$; Figure $3 \mathrm{c}$ and $3 d)$. Loads of $B$. heparinolyticus were significantly decreased in vaccinated cows at 9 DIM $(P<0.01$; Figure $3 d)$. Bacterial loads for all other species were similar 
a)

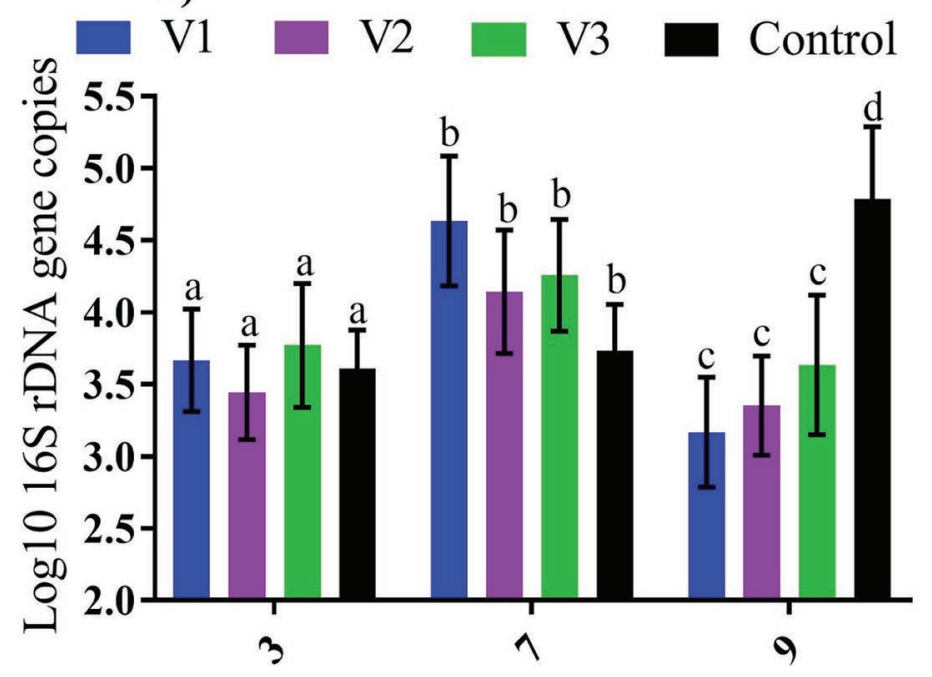

Days relative to parturition b)

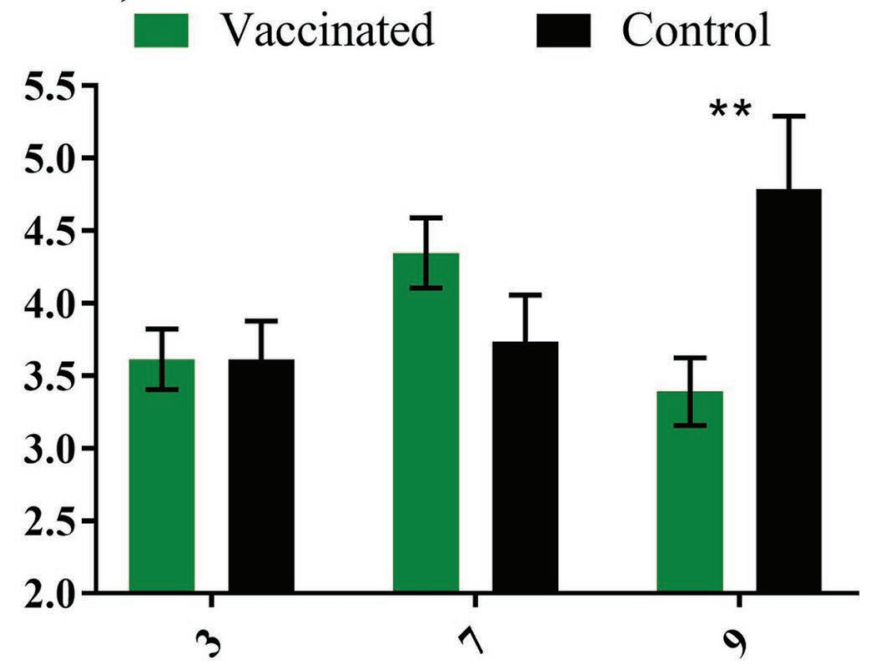

Days relative to parturition

Figure 2. Total bacterial load $\left(\log _{10} 16 \mathrm{~S}\right.$ rDNA gene copies) detected in vaginal samples $(\mathrm{n}=237$ swabs $)$ from postpartum dairy cows at 3 , 7, and 9 DIM presented as LSM \pm SEM. Graphs compare the effect of (a) individual vaccines (V) and (b) combined vaccines. In panel a, 1-way ANOVA test results are reported with the same letters denoting statistically similar values within each day $(P<0.05)$. Student's $t$-test results are reported for significant interaction terms $(* * P<0.01)$. The y-axis indicates the $\log _{10} 16 \mathrm{~S}$ rDNA copies of the estimated load for each species.

between vaccinated and control cows (Figure 3e). In general, most species' bacterial loads were highest at 7 and 9 DIM, with the exception of $U$. diversum, which was lowest at 7 DIM (Figure 3e).

\section{DISCUSSION}

Propelled by previous findings of vaccine efficacy by our research group (Machado et al., 2014), in the present study we evaluated the effect of 3 subcutaneous vaccine formulations on dairy cow uterine health. Similar to the results of Machado et al. (2014), we found that the subcutaneous vaccines as a group caused a reduction in the cumulative incidence of RDPM by 7 DIM when we applied a statistical contrast that compared the effect of vaccination, with any of the formulations, versus control. Furthermore, when we evaluated the effect of each vaccine separately, we identified a reduction in RDPM cumulative incidence with the recombinant subunit protein vaccine formulation (vaccine 3 ). Machado et al. (2014) were not able to find any effect of immunization on PVD prevalence using vaccine formulations similar to the ones we used here. Our results likewise suggest that immunization against the targeted antigens, while important for preventing puerperal metritis, were not effective in reducing the incidence of PVD.

Metritis is characterized by inflammation of the uterine wall, endometrium, and myometrium, and its severity may be assessed by the absence or presence of signs of systemic illness such as dullness, decreased milk yield, and fever (Sheldon et al., 2006). In the present study, vaccination reduced the cumulative incidence of severe uterine disease; however, we found no difference in the prevalence of $\mathrm{CM}$ (without fever). It has been reported that metritic cows that did not develop a fever had increased cure rates and improved fertility compared with metritic cows that developed a fever (Giuliodori et al., 2013; Lima et al., 2014). Cunha et al. (2018) suggested that the findings of increased cure rates and improved fertility for metritic cows that do not develop a fever indicated that the animals had been exposed to a lower bacterial challenge than the febrile metritic cows; however, the authors found no difference in TBL, so they then proposed that the febrile and nonfebrile cows mounted different immune responses to similar bacterial challenges. Demonstration of the benefits of vaccination on the clinical course of infections has been reported before (Deiss et al., 2015). Dosogne et al. (2002) proposed a potential mechanism by which E. coli J5 vaccine could protect cows against severe coliform mastitis: vaccine induction of tissue hyperresponsiveness characterized by a $\mathrm{T}$ helper 1 response and mediated by memory cells in the tissue, resulting in enhanced opsonization and neutrophil diapedesis upon intramammary infection. The result would be a more efficient response to tissue injury, causing a reduced bacterial load while modifying the inflammatory cascade that causes systemic illness. 
a)

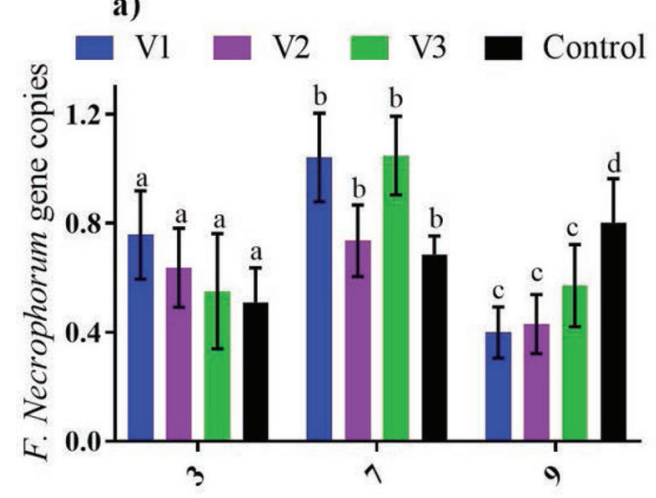

c)

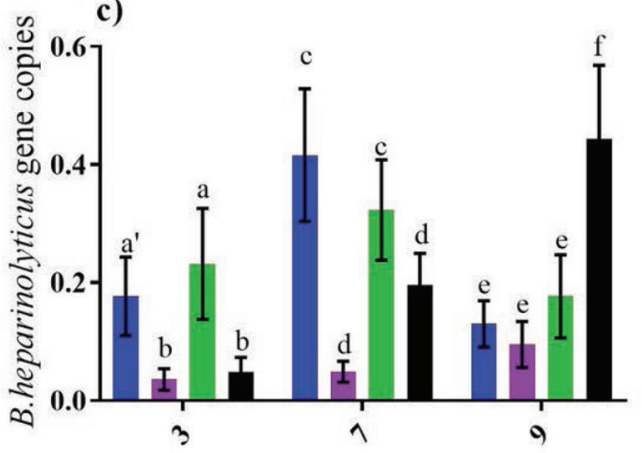

Days relative to parturition b)

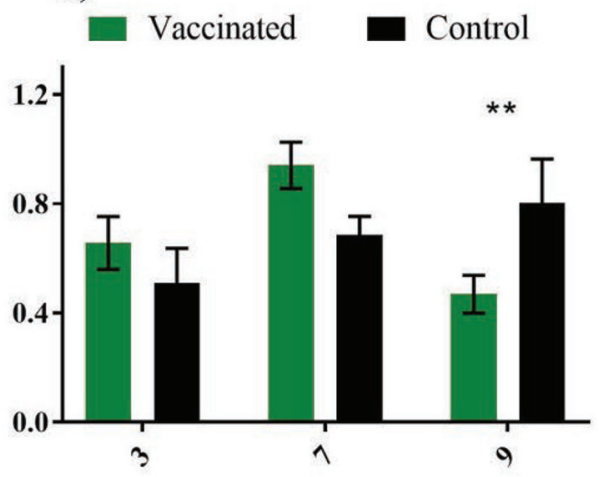

d)

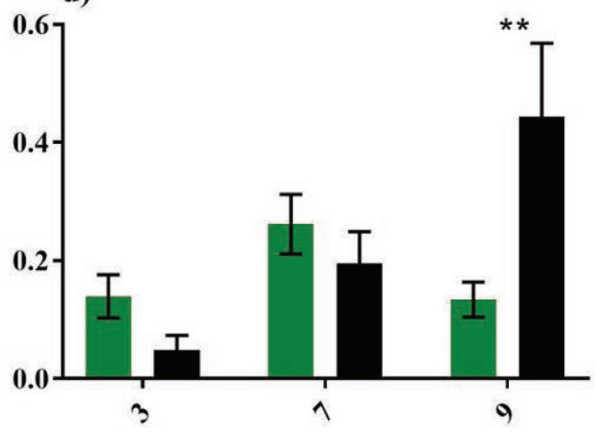

Days relative to parturition

e)

อ

$* \uparrow$ Fusobacterium necrophorum $\dagger$ Sneathia sanguinegens $\dagger$ Ureaplasma diversum Gallibacterium melopsittaci

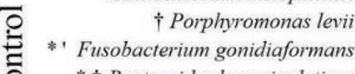

$* \dagger$ Bacteroides heparinolyticus $\uparrow$ Bacteroides pyogenes

$\uparrow$ Mycoplasma californicum

†' Streptococcus pluranimalium $\mapsto$ Helcococcus ovis

$\uparrow$ Bacteroides denticanum

Fusobacterium necrophorum Sneathia sanguinegens Ureaplasma diversum

ত্য

Gallibacterium melopsittaci Porphyromonas levii

Fusobacterium gonidiaformans

Bacteroides heparinolyticus Bacteroides pyogenes

Mycoplasma californicum Streptococcus pluranimalium Helcococcus ovis

Bacteroides denticanum

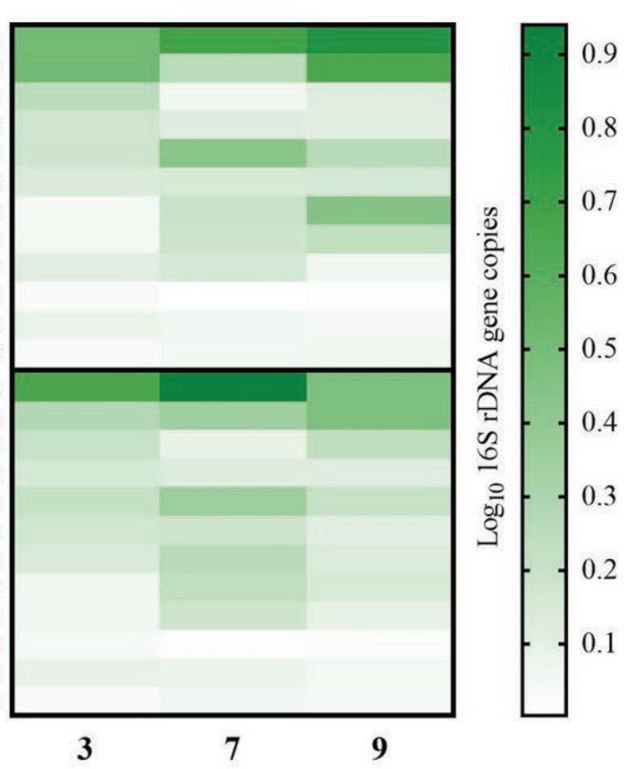

Days relative to parturition

Figure 3. Estimated species-specific bacterial loads ( $\log _{10} 16 \mathrm{~S}$ rDNA gene copies) of vaginal samples (n $=237$ swabs) from postpartum dairy cows at 3, 7, and 9 DIM are presented as LSM \pm SEM. The bacterial loads of (a and b) Fusobacterium necrophorum, and (c and d) Bacteroides heparinolyticus are compared for individual vaccines $(\mathrm{V})$ and combined vaccines. For individual vaccines, statistically similar values are noted $\left(P<0.05, P^{\prime}<0.10\right)$. For combined vaccines, the post-hoc Student $t$-test results are reported for significant interaction terms for each species $(* * P<0.01)$. The heatmap (e) displays $\log _{10} 16 \mathrm{~S}$ rDNA gene copies for the most abundant bacterial species divided into vaccinated and control cows at 3, 7, and 9 DIM. Darker shades indicate greater estimated bacterial load. For each species, significant fluctuations in bacterial load over time $(\dagger)$ and significant interactions between vaccination and time $(*)$ are noted $\left(P<0.05, P^{\prime}<0.10\right)$. The $\log _{10} 16 \mathrm{~S}$ rDNA copy number for each species was estimated by multiplying the $\log _{10}$ of the $16 \mathrm{~S}$ rDNA total bacterial copy number by the relative abundance for each species observed in the metagenomics results. 
Our results found that vaccinated cows had a lower TBL, specifically driven by a lower $F$. necrophorum abundance than control cows, and vaccinated cows had a higher likelihood of conceiving when compared with control cows, which suggests that vaccinated cows were able to produce a more effective immune response against uterine infection, with less exacerbated inflammation and associated tissue damage.

Metritis is known to be primarily associated with pathogenic gram-negative facultative and obligate anaerobes (Sheldon et al., 2009; Bicalho et al., 2012). Fusobacterium necrophorum is a gram-negative bacteria and has LPS present in its outer membrane, which is known to cause endotoxemia and systemic illness in cattle (Bannerman et al., 2003). The reduction of bacterial load decreases the severity of uterine disease (Sheldon et al., 2006). In the present study, we found that vaccinated cows had a significant reduction of their vaginal bacterial load, driven by decreases in loads of $F$. necrophorum and $B$. heparinolyticus. The effect of vaccination on the composition of the vaginal microbiome in postpartum cows was analyzed on a community-wide level at 3, 7, and 9 DIM. The Shannon index is a commonly used ecological diversity index that accounts for the total species richness (number of species) and species evenness (the equal distribution of community members) in a sample. Likewise, the Chao 1 index is an estimation of true species richness that estimates rare members in a community that may be missed due to undersampling. Both indexes help estimate the intrasample diversity of a microbial community. Therefore, in the postpartum vaginal biome, time had the most substantial effect on species diversity, which became reduced around $7 \mathrm{~d}$ postpartum. This is a challenging period for the immune system in a heifer's genital tract. There is increased microbial colonization of the genital tract by pathogenic bacteria, such as F. necrophorum, and a decrease in overall bacterial diversity. Accordingly, we observed the highest incidence of RDPM and FDPM at this time.

Machado et al. (2014), after vaccination, detected increased serum levels of IgG against E. coli, FimH, F. necrophorum, Lkt, T. pyogenes, and Plo. It has been reported that $F$. necrophorum Lkt exhibits high toxicity by inducing apoptosis-mediated killing of bovine polymorphonuclear neutrophils (Tan et al., 1992, 1994; Narayanan et al., 2002). Machado et al. (2014) theorized that immunizing cows against Lkt might have reduced the detrimental effect of this toxin on intrauterine polymorphonuclear neutrophils, thus improving the ability of the immune system to eliminate bacteria from the uterus through phagocytosis decrease the TBL. All the heifers in the study had been immunized with an $E$. coli O111:B4 (J5) bacterin, J-Vac (Merial); we cannot state if there was any interaction or cross-reaction to our vaccine. However, our results show that vaccinated cows had lower puerperal metritis prevalence and a sharp decrease in the estimated load of F. necrophorum, which may be attributable to an induced hyperresponsiveness of the reproductive mucosa caused by an Lkt vaccine effect.

An association between Bacteroides spp. and metritis has been reported in dairy cows (Jeon et al., 2016; Bicalho et al., 2017a; Cunha et al., 2018). Jeon et al. (2016) proposed that Bacteroides spp. are likely involved in the development of metritis. Not only does B. heparinolyticus possess multiple VF such as hemolysin, but it also has been shown to harbor antibiotic resistance genes resistant to ceftiofur (Jeon and Galvão, 2018). Our results show that vaccination targeting $F$. necrophorum and associated VF decreased the prevalence of puerperal metritis and the estimated load of Bacteroidetes. Price and McCallum (1986) reported that Bacteroides spp. thrive in the presence of a growth-stimulating factor secreted by F. necrophorum. Additionally, we noted that, in general, in postpartum cows, F. necrophorum relative abundance peaked at 7 DIM, and Bacteroidetes had higher estimated relative abundances in control cows at 9 DIM, suggesting that Bacteroidetes could thrive opportunistically in an F. necrophorum-conducive environment, and its contribution to the onset of metritis may be downregulated by immunization targeting $F$. necrophorum and its associated VF. Therefore, the fact that vaccination in our study reduced the bacterial load of $B$. heparinolyticus is a finding that warrants further exploration. One limitation of this study, is that we were only able to explore changes in TBL within the postpartum vaginal tract versus the uterus. Future studies must determine if the postpartum vaginal bacterial counts reported herein correlate with postpartum uterine bacterial loads. This study found that heifers vaccinated against $F$. necrophorum had a reduced vaginal bacterial load in the postpartum period, and this may have had a subsequent effect on the abundance of other species in the reproductive microbiome.

Consequently, prevalence of puerperal metritis was reduced in the postpartum period, which may be explained by globally altered interactions within the postpartum reproductive tract between mucosal immunity and the microbial community. Future studies are needed to determine the exact mechanisms by which vaccination induces changes to the bovine postpartum vaginal microbiome. To our knowledge, this is the first study in cattle to use quantitative next-generation genetic sequencing to demonstrate how bacterin vaccines can induce species-specific antibacterial effects on target bacterial species, thus reducing disease pressures in the target organs. 


\section{CONCLUSIONS}

The prevalence of puerperal metritis was significantly decreased by prepartum subcutaneous inoculation with vaccines containing bacterial VF proteins (FimH, Lkt, Plo) and inactivated whole cells (E. coli, F. necrophorum, and T. pyogenes). The vaccine formulation containing the VF subunits FimH, Lkt, and Plo was the most effective in reducing the prevalence of puerperal metritis. The vaccines effectively modulated the vaginal microbiome by decreasing the TBL and significantly suppressing one of their primary targeted microorganisms ( $F$. necrophorum), thereby fortifying the immune response to the postpartum challenge.

\section{ACKNOWLEDGMENTS}

The authors thank the collaborating dairy farm and its staff for providing the animals used in this study and their assistance during the project. This work was supported by Merck Animal Health (Madison, NJ). R. Linwood is an employee of Merck Animal Health (De Soto, KS). Vaccine production and formulation were conducted by Merck Animal Health (Madison, NJ). Other authors have not stated any conflict of interest.

\section{REFERENCES}

Aust, V., K. Knappstein, H. J. Kunz, H. Kaspar, J. Wallmann, and M. Kaske. 2013. Feeding untreated and pasteurized waste milk and bulk milk to calves: Effects on calf performance, health status and antibiotic resistance of faecal bacteria. J. Anim. Physiol. Anim. Nutr. (Berl.) 97:1091-1103. https://doi.org/10.1111/jpn.12019.

Azawi, O. I. 2008. Postpartum uterine infection in cattle. Anim. Reprod. Sci. 105:187-208. https://doi.org/10.1016/j.anireprosci.2008 .01 .010 .

Bannerman, D. D., M. J. Paape, W. R. Hare, and E. J. Sohn. 2003. Increased levels of LPS-binding protein in bovine blood and milk following bacterial lipopolysaccharide challenge. J. Dairy Sci. 86:3128-3137. https://doi.org/10.3168/jds.S0022-0302(03)73914 $-9$

Bicalho, M. L. S., V. S. Machado, C. H. Higgins, F. S. Lima, and R. C. Bicalho. 2017a. Genetic and functional analysis of the bovine uterine microbiota. Part I: Metritis versus healthy cows. J. Dairy Sci. 100:3850-3862. https://doi.org/10.3168/jds.2016-12058.

Bicalho, M. L. S., V. S. Machado, G. Oikonomou, R. O. Gilbert, and R. C. Bicalho. 2012. Association between virulence factors of Escherichia coli, Fusobacterium necrophorum, and Arcanobacterium pyogenes and uterine diseases of dairy cows. Vet. Microbiol. 157:125-131. https://doi.org/10.1016/j.vetmic.2011.11.034.

Bicalho, M. L. S., T. Santin, M. X. Rodrigues, C. E. Marques, S. F. Lima, and R. C. Bicalho. 2017b. Dynamics of the microbiota found in the vaginas of dairy cows during the transition period: Associations with uterine diseases and reproductive outcome. J. Dairy Sci. 100:3043-3058. https://doi.org/10.3168/jds.2016-11623.

Bicalho, R. C., V. S. Machado, M. L. Bicalho, R. O. Gilbert, A. G. Teixeira, L. S. Caixeta, and R. V. Pereira. 2010. Molecular and epidemiological characterization of bovine intrauterine Escherichia coli. J. Dairy Sci. 93:5818-5830. https://doi.org/10.3168/jds.2010 -3550 .

Brotman, R. M., J. Ravel, P. M. Bavoil, P. E. Gravitt, and K. G. Ghanem. 2014. Microbiome, sex hormones, and immune responses in the reproductive tract: Challenges for vaccine development against sexually transmitted infections. Vaccine 32:1543-1552. https://doi .org/10.1016/j.vaccine.2013.10.010.

Caporaso, J. G., C. L. Lauber, W. A. Walters, D. Berg-Lyons, J. Huntley, N. Fierer, S. M. Owens, J. Betley, L. Fraser, M. Bauer, N. Gormley, J. A. Gilbert, G. Smith, and R. Knight. 2012. Ultrahigh-throughput microbial community analysis on the Illumina HiSeq and MiSeq platforms. ISME J. 6:1621-1624. https://doi .org/10.1038/ismej.2012.8.

Cunha, F., S. J. Jeon, R. Daetz, A. Vieira-Neto, J. Laporta, K. C. Jeong, A. F. Barbet, C. A. Risco, and K. N. Galvao. 2018. Quantifying known and emerging uterine pathogens, and evaluating their association with metritis and fever in dairy cows. Theriogenology 114:25-33. https://doi.org/10.1016/j.theriogenology.2018.03.016.

Deiss, R. G., J. C. Arnold, W. J. Chen, S. Echols, M. P. Fairchok, C. Schofield, P. J. Danaher, E. McDonough, M. Ridore, D. Mor, T. H. Burgess, and E. V. Millar. 2015. Vaccine-associated reduction in symptom severity among patients with influenza A/H3N2 disease. Vaccine 33:7160-7167. https://doi.org/10.1016/j.vaccine 2015.11.004.

Dohmen, M. J., K. Joop, A. Sturk, P. E. Bols, and J. A. Lohuis. 2000. Relationship between intra-uterine bacterial contamination, endotoxin levels and the development of endometritis in postpartum cows with dystocia or retained placenta. Theriogenology 54:10191032. https://doi.org/10.1016/S0093-691X(00)00410-6.

Dolejska, M., Z. Jurcickova, I. Literak, L. Pokludova, J. Bures, A. Hera, L. Kohoutova, J. Smola, and A. Cizek. 2011. IncN plasmids carrying bla CTX-M-1 in Escherichia coli isolates on a dairy farm. Vet. Microbiol. 149:513-516. https://doi.org/10.1016/j.vetmic .2010.11.032.

Dosogne, H., F. Vangroenweghe, and C. Burvenich. 2002. Potential mechanism of action of $\mathrm{J} 5$ vaccine in protection against severe bovine coliform mastitis. Vet. Res. 33:1-12. https://doi.org/10.1051/ vetres:2001001.

Fricke, P. M., D. Z. Caraviello, K. A. Weigel, and M. L. Welle. 2003. Fertility of dairy cows after resynchronization of ovulation at three intervals following first timed insemination. J. Dairy Sci. 86:39413950. https://doi.org/10.3168/jds.S0022-0302(03)74003-X.

Gilbert, J. A., F. Meyer, D. Antonopoulos, P. Balaji, C. T. Brown, C. T. Brown, N. Desai, J. A. Eisen, D. Evers, D. Field, W. Feng, D. Huson, J. Jansson, R. Knight, J. Knight, E. Kolker, K. Konstantindis, J. Kostka, N. Kyrpides, R. Mackelprang, A. McHardy, C. Quince, J. Raes, A. Sczyrba, A. Shade, and R. Stevens. 2010. Meeting report: The terabase metagenomics workshop and the vision of an Earth microbiome project. Stand. Genomic Sci. 3:243248. https://doi.org/10.4056/sigs.1433550.

Giuliodori, M. J., R. P. Magnasco, D. Becu-Villalobos, I. M. LacauMengido, C. A. Risco, and R. L. de la Sota. 2013. Metritis in dairy cows: Risk factors and reproductive performance. J. Dairy Sci. 96:3621-3631. https://doi.org/10.3168/jds.2012-5922.

Jeon, S. J., F. Cunha, X. Ma, N. Martinez, A. Vieira-Neto, R. Daetz, R. C. Bicalho, S. Lima, J. E. Santos, K. C. Jeong, and K. N. Galvao. 2016. Uterine microbiota and immune parameters associated with fever in dairy cows with metritis. PLoS One 11:e0165740. https://doi.org/10.1371/journal.pone.0165740.

Jeon, S. J., and K. N. Galvão. 2018. An advanced understanding of uterine microbial ecology associated with metritis in dairy cows. Genomics Inform. 16:e21. https://doi.org/10.5808/GI.2018.16.4 .e21.

Kelton, D. F., K. D. Lissemore, and R. E. Martin. 1998. Recommendations for recording and calculating the incidence of selected clinical diseases of dairy cattle. J. Dairy Sci. 81:2502-2509. https://doi .org/10.3168/jds.S0022-0302(98)70142-0.

Lima, F. S., A. Vieira-Neto, G. S. Vasconcellos, R. D. Mingoti, E. Karakaya, E. Sole, R. S. Bisinotto, N. Martinez, C. A. Risco, K. N. Galvao, and J. E. Santos. 2014. Efficacy of ampicillin trihydrate or ceftiofur hydrochloride for treatment of metritis and subsequent fertility in dairy cows. J. Dairy Sci. 97:5401-5414. https://doi.org/ 10.3168/jds.2013-7569.

Machado, V. S., M. L. Bicalho, E. B. Meira Junior, R. Rossi, B. L. Ribeiro, S. Lima, T. Santos, A. Kussler, C. Foditsch, E. K. Ganda, G. Oikonomou, S. H. Cheong, R. O. Gilbert, and R. C. Bicalho. 
2014. Subcutaneous immunization with inactivated bacterial components and purified protein of Escherichia coli, Fusobacterium necrophorum and Trueperella pyogenes prevents puerperal metritis in Holstein dairy cows. PLoS One 9:e91734. https://doi.org/10 .1371/journal.pone.0091734.

Moreira, F., C. Orlandi, C. A. Risco, R. Mattos, F. Lopes, and W. W. Thatcher. 2001. Effects of presynchronization and bovine somatotropin on pregnancy rates to a timed artificial insemination protocol in lactating dairy cows. J. Dairy Sci. 84:1646-1659. https: //doi.org/10.3168/jds.S0022-0302(01)74600-0.

Narayanan, S., G. C. Stewart, M. M. Chengappa, L. Willard, W. Shuman, M. Wilkerson, and T. G. Nagaraja. 2002. Fusobacterium necrophorum leukotoxin induces activation and apoptosis of bovine leukocytes. Infect. Immun. 70:4609-4620. https://doi.org/10 $.1128 /$ IAI.70.8.4609-4620.2002.

Overton, M., and J. Fetrow. 2008. Economics of postpartum uterine health. Dairy Cattle Reproduction Council Convention, Omaha, NE. November 7-8, 2008.

Price, S. B., and R. E. McCallum. 1986. Enhancement of Bacteroides intermedius growth by Fusobacterium necrophorum. J. Clin. Microbiol. 23:22-28. https://doi.org/10.1128/JCM.23.1.22-28.1986.

Pursley, J. R., M. O. Mee, and M. C. Wiltbank. 1995. Synchronization of ovulation in dairy cows using PGF2alpha and GnRH. Theriogenology 44:915-923. https://doi.org/10.1016/0093 $-691 \mathrm{X}(95) 00279-\mathrm{H}$.

Santos, T. M., L. S. Caixeta, V. S. Machado, A. K. Rauf, R. O. Gilbert, and R. C. Bicalho. 2010. Antimicrobial resistance and presence of virulence factor genes in Arcanobacterium pyogenes isolated from the uterus of postpartum dairy cows. Vet. Microbiol. 145:8489. https://doi.org/10.1016/j.vetmic.2010.03.001.

Sheldon, I. M., J. Cronin, L. Goetze, G. Donofrio, and H. J. Schuberth. 2009. Defining postpartum uterine disease and the mechanisms of infection and immunity in the female reproductive tract in cattle. Biol. Reprod. 81:1025-1032. https://doi.org/10.1095/biolreprod .109.077370.

Sheldon, I. M., G. S. Lewis, S. LeBlanc, and R. O. Gilbert. 2006. Defining postpartum uterine disease in cattle. Theriogenology 65:1516-1530. https://doi.org/10.1016/j.theriogenology.2005.08 .021 .

Sheldon, I. M., E. J. Williams, A. N. Miller, D. M. Nash, and S. Herath. 2008. Uterine diseases in cattle after parturition. Vet. J. 176:115-121. https://doi.org/10.1016/j.tvjl.2007.12.031.

Tan, Z. L., T. G. Nagaraja, and M. M. Chengappa. 1992. Factors affecting the leukotoxin activity of Fusobacterium necrophorum. Vet. Microbiol. 32:15-28. https://doi.org/10.1016/0378-1135(92)90003 $-\mathrm{C}$.

Tan, Z. L., T. G. Nagaraja, M. M. Chengappa, and J. S. Smith. 1994. Biological and biochemical characterization of Fusobacterium necrophorum leukotoxin. Am. J. Vet. Res. 55:515-521.

Valdez, Y., E. M. Brown, and B. B. Finlay. 2014. Influence of the microbiota on vaccine effectiveness. Trends Immunol. 35:526-537. https://doi.org/10.1016/j.it.2014.07.003.

Vieira-Neto, A., F. Lima, J. Santos, R. Mingoti, G. Vasconcellos, C. Risco, and K. Galvao. 2016. Vulvovaginal laceration as a risk factor for uterine disease in postpartum dairy cows. J. Dairy Sci. 99:4629-4637. https://doi.org/10.3168/jds.2016-10872. 